\title{
Environmental Control of Charge Density Wave Order in Monolayer $2 \mathrm{H}-\mathrm{TaS}_{2}$
}

Joshua Hall, ${ }^{*, \dagger}$ Niels Ehlen, ${ }^{\dagger}$ Jan Berges, ${ }^{\ddagger}$ Erik van Loon, ${ }^{\ddagger}$ Camiel van Efferen, ${ }^{\dagger}$ Clifford Murray, $^{\dagger}$ Malte Rösner, ${ }^{\top}$ Jun Li, ${ }^{\dagger}$ Boris V. Senkovskiy, ${ }^{\dagger}$ Martin Hell, $^{\dagger}$ Matthias Rolf, ${ }^{\dagger}$ Tristan Heider, ${ }^{\S}$ María C. Asensio," José Avila," Lukasz Plucinski, ${ }^{\S}$ Tim Wehling, ${ }^{\ddagger}$ Alexander Grüneis, ${ }^{\dagger}$ and Thomas Michely ${ }^{\dagger}$

†II. Physikalisches Institut, Universität zu Köln, Zülpicher Straße 77, 50937 Köln, Germany

$\ddagger$ Institut für Theoretische Physik, Bremen Center for Computational Materials Science, Universität Bremen, Otto-Hahn-Allee 1, 28359 Bremen, Germany

IInstitute for Molecules and Materials, Radboud University, 6525 AJ Nijmegen, The Netherlands

§Peter Grünberg Institut (PGI-6), Forschungszentrum Jülich GmbH, 52425 Jülich, Germany

\|ANTARES Beamline, Synchrotron SOLEIL and Universite Paris-Saclay, L' Orme des Merisiers, Saint Aubin-BP 48, 91192 Gif sur Yvette Cedex, France

E-mail: hall@ph2.uni-koeln.de

\begin{abstract}
For quasi-freestanding $2 \mathrm{H}-\mathrm{TaS}_{2}$ in monolayer thickness grown by in situ molecular beam epitaxy on graphene on $\operatorname{Ir}(111)$, we find unambiguous evidence for a charge density wave close to a $3 \times 3$ periodicity. Using scanning tunneling spectroscopy, we
\end{abstract}


determine the magnitude of the partial charge density wave gap. Angle-resolved photoemission spectroscopy, complemented by scanning tunneling spectroscopy for the unoccupied states, makes a tight-binding fit for the band structure of the $\mathrm{TaS}_{2}$ monolayer possible. As hybridization with substrate bands is absent, the fit yields a precise value for the doping of the $\mathrm{TaS}_{2}$ layer. Additional Li doping shifts the charge density wave to a $2 \times 2$ periodicity. Unexpectedly, the bilayer of $\mathrm{TaS}_{2}$ also displays a disordered $2 \times 2$ charge density wave. Calculations of the phonon dispersions based on a combination of density-functional theory, density-functional perturbation theory, and many-body perturbation theory enable us to provide phase diagrams for the $\mathrm{TaS}_{2}$ charge density wave as functions of doping, hybridization and interlayer potentials, and offer insight into how they affect lattice dynamics and stability. Our theoretical considerations are consistent with the experimental work presented and shed light on previous experimental and theoretical investigations of related systems.

\section{Keywords}

transition metal dichalcogenides, $\mathrm{TaS}_{2}$, monolayer, charge density wave, layer dependence, doping, hybridization

Condensed matter quantum many-body states are often highly sensitive to stimuli such as pressure, temperature, or changes in chemical composition. Therefore, the concurrence of pronounced many-body phenomena in (quasi-) two-dimensional (2d) materials ${ }^{1-4}$ with advances in synthesis and vertical heterostructuring ${ }^{5,6}$ has fueled hopes for controlling electronic quantum phases on demand. ${ }^{7}$ These hopes are supported by experiments revealing electronic phase diagrams of several $2 \mathrm{~d}$ systems - including Fe-based superconductors ${ }^{8}$ and transition metal dichalcogenides (TMDCs) ${ }^{9-15}$ - to be strongly dependent on dimensionality, thickness, and substrate. However, the microscopic mechanisms behind these dependencies often remain elusive and a thorough understanding of how to tune electronic quantum phases by atomic scale manipulations is largely lacking to date - although being an inevitable re- 
quirement for implementation of quantum materials into device applications.

Using the example of the prototypical charge density wave (CDW) material $\mathrm{TaS}_{2}$, we experimentally and theoretically explore how CDW order is influenced by the control parameters doping and hybridization with the substrate. Regarding the latter, this is investigated in monolayer (ML) $\mathrm{TaS}_{2}$ as well as the case of interlayer interaction in bilayer (BL) $\mathrm{TaS}_{2}$. These parameters add additional dimensions to the phase diagram of $\mathrm{TaS}_{2}$ and allow for quantum phase transitions between different kinds of CDW ordered and distorted states. Based on theoretical modeling, we identify doping and hybridization driven phonon self-energy effects as the microscopic origins of the CDW transitions in the ML.

The material under consideration in the present study is the $2 \mathrm{H}$ polytype of $\mathrm{TaS}_{2}$, where the metal atom coordination is trigonal prismatic. As bulk material, it displays a slightly discommensurate $3 \times 3 \mathrm{CDW}^{16}$ phase with a partial gap, ${ }^{17-20}$ a CDW transition temperature of $75 \mathrm{~K},{ }^{21}$ and a transition to a superconducting phase at around $1 \mathrm{~K} .{ }^{22}$

In line with related materials, ${ }^{9,11,12,23}$ the electronic phase diagram of $2 \mathrm{H}-\mathrm{TaS}_{2}$ appears highly layer dependent: when approaching monolayer thickness, the superconducting transition temperature of $\mathrm{TaS}_{2}$ becomes enhanced as compared to the bulk. ${ }^{13-15,24,25} \mathrm{CDW}$ order turns out to depend on the number of layers too, but the monolayer limit remains unclear and controversial until now.

Few experimental works addressed charge order in ML $2 \mathrm{H}-\mathrm{TaS}_{2}$ recently. Sanders et al. ${ }^{26}$ found no $\mathrm{CDW}$ when a ML was grown on $\mathrm{Au}(111)$ by molecular beam epitaxy (MBE). Also, Yang et al. ${ }^{14}$ conclude the CDW for ML $2 \mathrm{H}-\mathrm{TaS}_{2}$ encapsulated in hexagonal boron nitride to be absent, based on transport measurements. In contrast, Lin et al. ${ }^{27}$ observed a $3 \times 3$ superstructure indicative of a CDW for the MBE grown ML on graphene (Gr) grown on $6 \mathrm{H}-\mathrm{SiC}(0001)$. The situation is puzzling and stimulated several theoretical contributions. Freestanding undoped ML $2 \mathrm{H}-\mathrm{TaS}_{2}$ turns out to be dynamically unstable ${ }^{27,28}$ and favors CDW order. Further density functional theory studies show that interaction with substrates affects the tendency of ML $2 \mathrm{H}-\mathrm{TaS}_{2}$ towards CDW formation. ${ }^{28,29}$ 
For a doping level consistent with that derived from angle-resolved photoemission of ML $2 \mathrm{H}-\mathrm{TaS}_{2}$ on $\mathrm{Au}(111),{ }^{26}$ Albertini et al. ${ }^{28}$ find the CDW to be suppressed. Shao et al. ${ }^{30}$ point out that an estimate of the doping level in the $\mathrm{ML} 2 \mathrm{H}-\mathrm{TaS}_{2}$ on $\mathrm{Au}(111)$ based on changes in the Fermi surface (FS) may severely overestimate the actual charge transfer through non-linear band distortions as a consequence of hybridization. Correspondingly, LefcochilosFogelquist et al. ${ }^{29}$ argue that the suppression of the CDW in $\mathrm{ML} 2 \mathrm{H}-\mathrm{TaS}_{2}$ on $\mathrm{Au}(111)$ is primarily a consequence of hybridization in consequence of strong S-Au interactions, rather than usual charge doping. However, a clear disentanglement of hybridization and doping effects on CDW order is currently lacking.

Here, we take a close look at this issue and analyze experimentally the absence/presence of CDWs in quasi-freestanding ML $2 \mathrm{H}-\mathrm{TaS}_{2}$ at different doping levels, as well as the situation in the $\mathrm{BL}$ of $2 \mathrm{H}-\mathrm{TaS}_{2}$. Our theoretical analysis is not only fully consistent with the results of our experiments but also resolves apparent discrepancies in the experimental and theoretical works by clarifying the roles of hybridization, doping and interlayer interaction for lattice (de-)stabilization and the formation of CDW states.

\section{Results/Discussion}

\section{Pristine Monolayer $\mathrm{TaS}_{2}$}

Fig. 1 (a) displays a large scale STM topograph of MBE grown $\mathrm{TaS}_{2}$ on $\mathrm{Gr} / \mathrm{Ir}(111)$ taken at $5 \mathrm{~K}$. The geometrical coverage of 0.7 layers of $\mathrm{TaS}_{2}$ arranges in a network which covers about $65 \%$ of the $\operatorname{Gr} / \operatorname{Ir}(111)$ substrate and readily overgrows a step edge visible in the bottom part of the image. This network is decorated with about $5 \%$ coverage of small triangular islands in the second layer. As visible in the line profile along the black line in the topograph, the apparent STM height of ML $\mathrm{TaS}_{2}$ is $6.5 \AA$. This compares well to both the interlayer distance measured in bulk $2 \mathrm{H}-\mathrm{TaS}_{2}$ of $6.26 \AA,^{31,32}$ and to our density functional theory calculations (see Methods). 

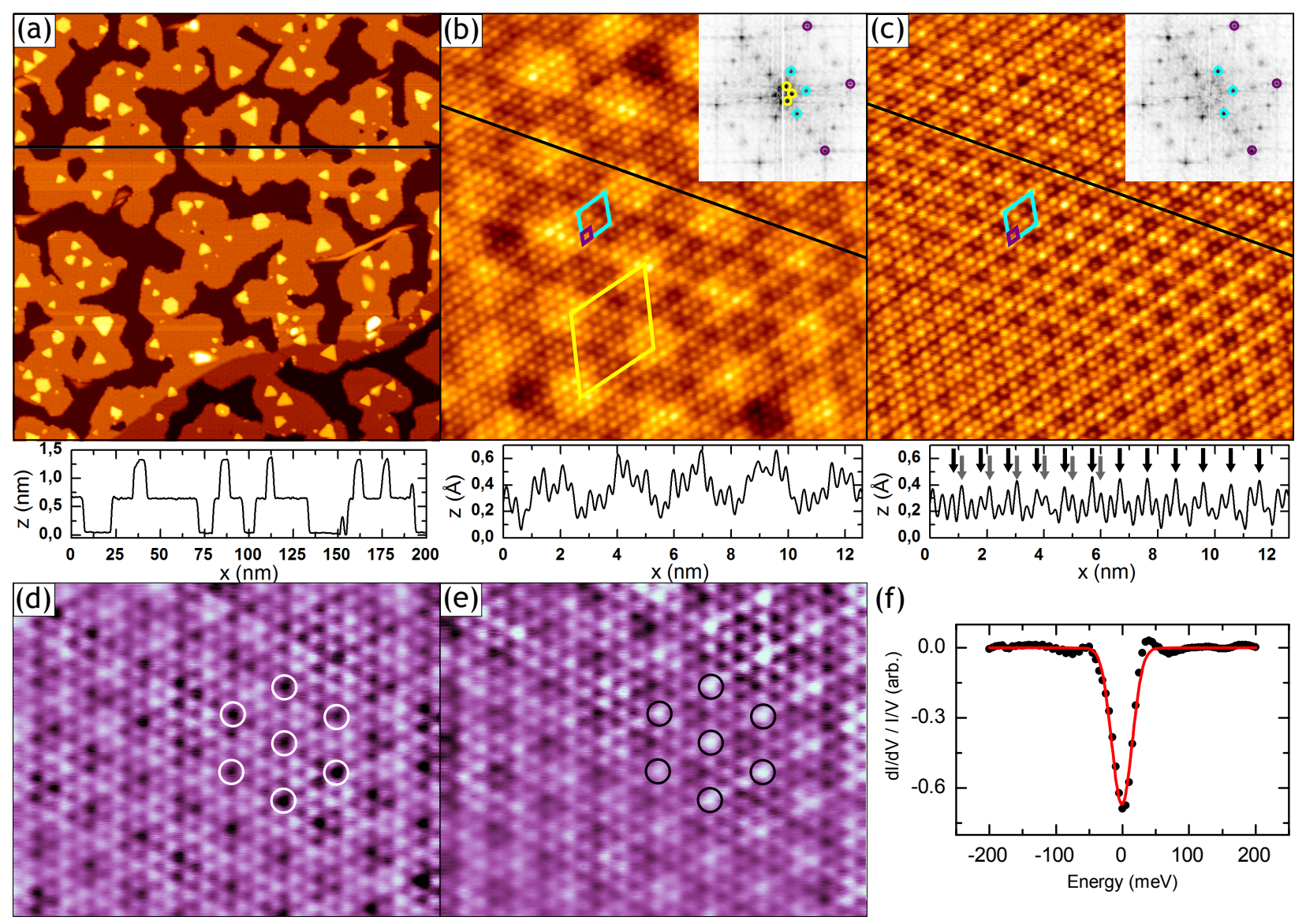

Figure 1: STM overview of the $\mathrm{TaS}_{2} / \mathrm{Gr} / \mathrm{Ir}(111)$ sample: (a) Large scale STM topograph of $\mathrm{TaS}_{2}$ islands on $\mathrm{Gr} / \mathrm{Ir}(111)$. Sample largely covered with $\mathrm{ML} \mathrm{TaS}_{2}$, on which mostly triangular second layer islands sit. On the right, a Gr wrinkle is visible, at the bottom right a step edge. The black line shows the position of the line profile at the bottom. (b) Constant current STM atomic resolution topograph of ML $\mathrm{TaS}_{2}$. Three periodic structures are indicated, each with its respective unit cell $(\mathrm{Gr} / \operatorname{Ir}(111)$ moiré yellow, $3 \times 3 \mathrm{CDW}$ turquoise, atomic $\mathrm{TaS}_{2}$ lattice violet). Inset: Fourier transform of the STM image with peaks related to the periodicities circled in same the color as unit cell rhomboids. (c) Same image as (b), but with the moiré filtered out in Fourier space to enhance the visibility of the CDW. Inset: Fourier transform of the STM image. The black line shows the position of the line profile at the bottom, where arrows denote the position of the local charge density maximum (see

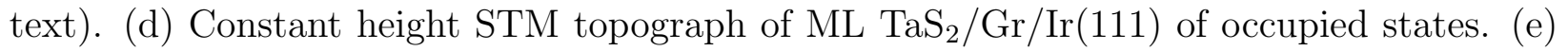
Corresponding constant height STM topograph of unoccupied states. The circles mark the same positions but either show local maxima or minima of the CDW. (f) Constant height tunneling spectrum on $\mathrm{ML} \mathrm{TaS}$, treated as in Ref. 33. The scale is adjusted to be comparable to Fig. 3 (f) and Fig. 4 (b).

Image information [image size, (stabilization) sample bias, (stabilization) tunneling current]: (a) $200 \times 200 \mathrm{~nm}^{2},-1 \mathrm{~V}, 0.1 \mathrm{nA},\left(\right.$ b), (c) $12 \times 12 \mathrm{~nm}^{2},-0.15 \mathrm{~V}, 0.2 \mathrm{nA}$, (d) $7 \times 5 \mathrm{~nm}^{2}$, $-0.02 \mathrm{~V}, I_{\text {stab }}=0.1 \mathrm{nA},(\mathrm{e}) 7 \times 5 \mathrm{~nm}^{2}, 0.02 \mathrm{~V}, I_{\text {stab }}=0.1 \mathrm{nA},(\mathrm{f}) U_{\text {stab }}=-0.3 \mathrm{~V}$, $I_{\text {stab }}=0.5 \mathrm{nA}$. 
In Fig. 1 (b), a close-up of the ML $\mathrm{TaS}_{2}$ surface is shown, along with its Fourier transform in the inset. The surface displays three different periodic structures, each being indicated with its respective unit cell in the STM and its corresponding Fourier peak in the inset, respectively.

The largest structure (yellow rhomboid) in the topograph is the moiré formed by $\mathrm{Gr} / \mathrm{Ir}(111)$ which is also visible in the $\mathrm{TaS}_{2}$ layer. Motivated by previous studies on similar TMDC/Gr/Ir(111) systems ${ }^{34,35}$ we interpret the lack of an additional moiré between $\mathrm{TaS}_{2}$ and $\mathrm{Gr}$ as a sign for the weak van der Waals (vdW) interaction of the TMDC with its substrate $\mathrm{Gr} / \operatorname{Ir}(111)$.

Due to the low interaction with the substrate, the $\mathrm{TaS}_{2}$ islands are not strictly epitaxial. Despite a preferential alignment of the dense-packed $\mathrm{TaS}_{2}$ and $\mathrm{Gr}$ rows, orientation scatter is present. For example, in the STM topograph shown in Fig. 1 (b) the orientation mismatch amounts to $4^{\circ}$.

The smallest structure (violet rhomboid) is the atomic lattice of $\mathrm{TaS}_{2}$, which through comparison with the moiré can be determined to be $(3.37 \pm 0.02) \AA$. It matches well with the bulk in-plane lattice constant found in the literature. ${ }^{31,32}$ This agreement is consistent with Raman experiments on very similar TMDC/Gr/Ir(111) systems. The investigations have shown that the TMDC layer follows its pristine thermal expansion without indication of strain even after high temperature annealing. ${ }^{36}$ Therefore, strain is not considered in the following.

The remaining middle-sized periodic structure can be identified as a $3 \times 3$ superstructure with respect to the atomic $\mathrm{TaS}_{2}$ lattice by filtering out the moiré, which is illustrated in Fig. 1 (c). The respective unit cells of the atomic lattice and the $3 \times 3$ superstructure are indicated in violet and turquoise respectively. In analogy to the bulk counterpart, we associate this $3 \times 3$ superstructure with a CDW phase of $\mathrm{ML} \mathrm{TaS}_{2}$ found at sufficiently low temperatures, and will characterize some of its fingerprints in the following.

It is hard to see the details of the $3 \times 3 \mathrm{CDW}$ from the line profile in Fig. 1 (b), as the profile is dominated by the moiré corrugation of about $0.5 \AA$. Removing the moiré in 
Fig. 1 (c), allows examination of spatial details of the CDW. Following the black arrows (marking the position of the highest atom in the line profile) from right to left reveals the $3 a$ periodicity, where $a$ is the lattice constant of $\mathrm{TaS}_{2}$. However, on the left-hand side of the line profile, the $3 a$ periodicity of the highest atoms is drawn by the position of the grey arrows, which are shifted relative to the black arrows. This indicates a phase shift in the CDW consistent with the absence of strict commensurability. ${ }^{16}$ More details on the interaction of the CDW with island terminations and defects are found in the Supporting Information.

In order to demonstrate the CDW origin of the superstructure, we probe states above and below the Fermi energy by constant height STM and show results in Fig. 1 (d) and (e). A contrast inversion takes place upon change of polarity, as highlighted by the black circles in (d) enclosing brightness maxima and the white circles in (e) enclosing brightness minima. Both sets of circles are located around the same atoms. In a CDW, the occupied and unoccupied states above and below the Fermi energy have the same wave vector $k_{\mathrm{F}}$ but different energies, resulting in antiphase behavior in real space. This behavior is clearly reproduced in the respective figures.

Performing scanning tunneling spectroscopy (STS) at $5 \mathrm{~K}$ reveals a reduction of the local density of states (LDOS) at the Fermi energy [cf. Fig. 1 (f)], which (to our best knowledge) has not been reported before for $\mathrm{ML} \mathrm{TaS}_{2}$. We associate this feature with partial gapping of the FS similar to the bulk case. Following the methodology of Ref. 33, we divide the $d I / d V$ signal by $I / V$ whereby the features in the LDOS become more pronounced (see Supporting Information). After background subtraction, we find a width of $2 \Delta=(32 \pm 9) \mathrm{meV}$, where the scatter reflects the variation of the measured CDW gap due to variations of the tip state. Compared to the data of bulk $2 \mathrm{H}-\mathrm{TaS}_{2}$ in which $2 \Delta=100 \mathrm{meV},{ }^{18,19}$ our gap is significantly smaller. To elaborate on this, we note that in previous work the gap size was merely estimated, while we use a reproducible method described in Ref. 33, which may contribute substantially to the difference. Ref. 33 points out that in STS on TMDCs the partial CDW gap may be located very near to features related to the flat $d$ band of the transition metal. 
These may be assigned falsely to the CDW gap edge and artificially increase the gap size. The normalization by $I / V$ helps to disentangle these effects and puts the gap determination on reproducible grounds.

In Fig. 2 (a), angle-resolved photoemission spectroscopy (ARPES) scans in high symmetry directions are shown. The ARPES data indicate a spin-orbit split band in the energy range close to the Fermi level. It is attributed to the Ta $d$-states, based on literature data for the $2 \mathrm{H}$ polytype. ${ }^{20,37,38}$ The band is composed of a nearly degenerate hole-like pocket around $\Gamma$ and of spin-split hole-like pockets at the inequivalent $\mathrm{K}$ points. Discrete values for the dispersion obtained by standing wave pattern analysis of STS data around $\Gamma$ are shown as blue dots (see also Supporting Information).

Fig. 2 (b) displays the FS of $\mathrm{TaS}_{2} / \mathrm{Gr} / \mathrm{Ir}(111)$ measured by ARPES. The hexagonal hole pocket around the center of the Brillouin zone and the spin-split hole pockets around the K-points compare qualitatively well with bulk ARPES results. ${ }^{37,38}$

We performed a tight-binding (TB) fit of the experimental band structure taking into account all measured experimental ARPES and STS data and treating all TB parameters as mere fit parameters. The fit is represented in Figs. 2 (a) and (b) as lines ranging from yellow to blue in dependence of their spin character. By comparison to the TB fit, the spin-orbit split nature of the band in the $\Gamma K$-direction and of the hole-like pockets at the inequivalent K-points become obvious. Overall, the TB fit perfectly reproduces the observed spectra, and the ARPES data display no hybridization between the $\mathrm{TaS}_{2}$ adlayer and its substrate.

From the relative area of the occupied states enclosed by the tight-binding fitted FS, we calculate the free charge carrier concentration and find $1.10 \pm 0.02$ electrons per unit cell, that is an excess or doping of 0.1 electrons per unit cell (in the following denoted as $x=-0.10$ ) in comparison to half-filling of the band (for details see Methods). As the TB band structure is a fit to the experimental band structure, the estimate of the doping level based on it is much more precise than an estimate based on ab initio calculations. 
(a)

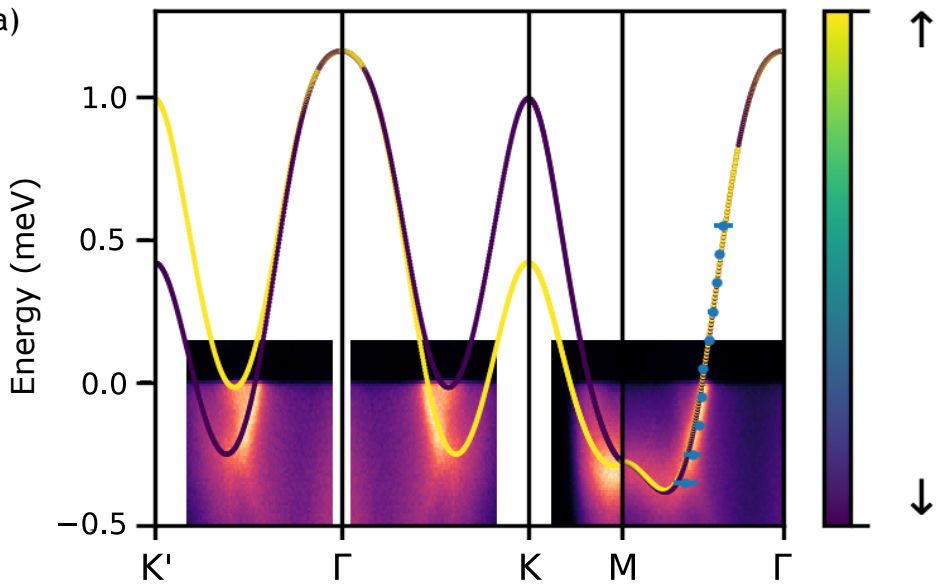

(b)

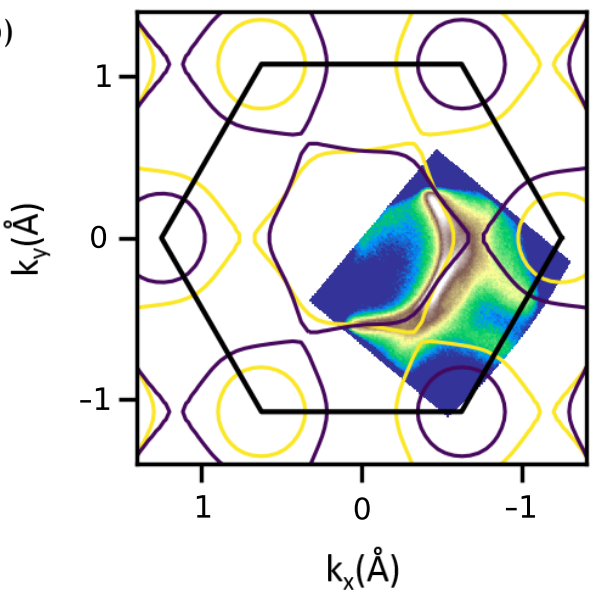

Figure 2: Band structure of $\mathrm{ML} \mathrm{TaS}_{2}$ : (a) Tight-binding fit of the band structure based on experimental ARPES and STM (blue dots) data. The color of the calculated bands indicates the spin contribution; yellow: spin up, magenta: spin down. The color scale of the ARPES spectra in the background indicates photoemission intensity from orange (high intensity) to violet (low intensity). (b) Fermi surface as measured with ARPES and tight-binding fit of FS. Color scale of the calculated bands as in (a), color scale of the FS ARPES data in the background indicates photoemission intensity from white (high intensity) to blue (low intensity). ARPES data was taken at about $40 \mathrm{~K}$ using $21 \mathrm{eV}$ photon energy, with the exception of the $K M$ direction which was taken at about $100 \mathrm{~K}$ and $50 \mathrm{eV}$ photon energy.

In summary, we find slightly doped $\mathrm{ML} \mathrm{TaS}_{2}$ to show a CDW state, with a periodicity close to $3 \times 3$. Both the CDW periodicity and the TB band structure we obtain from ARPES and STS are similar to the bulk counterpart.

\section{Bilayer $\mathrm{TaS}_{2}$}

In Fig. 3 (a), a large scale STM overview of a sample with substantial BL coverage is shown. The top layer islands grow perfectly aligned to the respective ML TaS $\mathrm{T}_{2}$ bottom layer on $\mathrm{Gr} / \mathrm{Ir}(111)$. We never find the second layer of $\mathrm{TaS}_{2}$ with a rotational misalignment relative to the bottom layer. The strict epitaxial relation of the two $\mathrm{TaS}_{2}$ layers is interpreted as a consequence of a strong interaction between the two layers in the BL case; the absence of the $\mathrm{Gr} / \mathrm{Ir}(111)$ moiré in BL islands is consistent with a stronger interaction of the two $\mathrm{TaS}_{2}$ layers as compared to the $\mathrm{Gr}-\mathrm{TaS}_{2}$ interaction.

Fig. 3 (b) shows one of the BL islands in higher resolution which exhibits internal struc- 

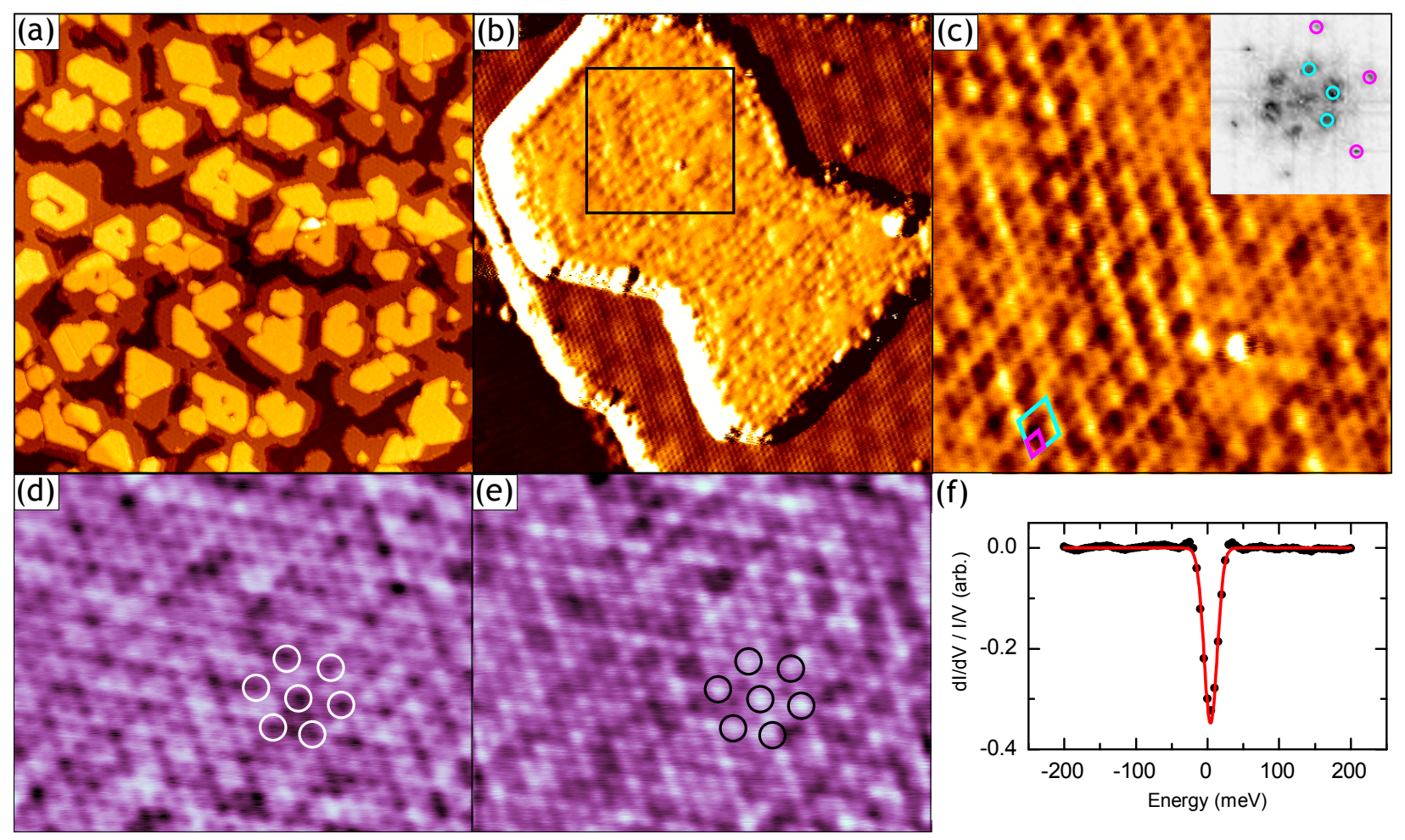

(f)

Figure 3: STM overview of $\mathrm{BL} \mathrm{TaS}_{2} / \mathrm{Gr} / \mathrm{Ir}(111)$. (a) STM overview topograph. (b) Close up of $\mathrm{BL} \mathrm{TaS}_{2}$ showing a disturbed $2 \times 2$ superstructure. The black square indicates the location of panel (c). (c) Constant current STM showing the $2 \times 2$ superstructure with atomic resolution on $\mathrm{BL} \mathrm{TaS}$. The respective unit cells $\left(2 \times 2 \mathrm{CDW}\right.$ turquoise, atomic $\mathrm{TaS}_{2}$ lattice violet) are shown. Inset: Fourier transform of the STM image with peaks related to the periodicity represented by unit cells circled in same color as unit cell rhomboids. (d) Constant height STM topograph of the occupied states. (e) Corresponding constant height STM topograph of unoccupied states. The circles mark the same positions but indicate either local maxima or minima of the CDW. (f) normalized constant height STS spectrum on BL $\mathrm{TaS}_{2} .{ }^{33}$ The scale is adjusted to be comparable to Fig. 1 (f) and Fig. 4 (b).

Image information [image size, (stabilization) sample bias, (stabilization) tunneling current]: (a) $200 \times 200 \mathrm{~nm}^{2}, 1.5 \mathrm{~V}, 0.1 \mathrm{nA}$, (b) $25 \times 25 \mathrm{~nm}^{2},-1 \mathrm{~V}, 1 \mathrm{nA}$, (c) $8 \times 8 \mathrm{~nm}^{2}$, $-1 \mathrm{~V}, 4 \mathrm{nA}(\mathrm{d}) 7 \times 5 \mathrm{~nm}^{2},-0.05 \mathrm{~V}, I_{\text {stab }}=0.1 \mathrm{nA}$, (e) $7 \times 5 \mathrm{~nm}^{2}, 0.05 \mathrm{~V}, I_{\text {stab }}=0.1 \mathrm{nA}$, (f) $U_{\text {stab }}=-0.2 \mathrm{~V}, I_{\text {stab }}=10 \mathrm{pA}$. 
ture. This is found to be a $2 \times 2$ superstructure with respect to the atomic lattice of $\mathrm{TaS}_{2}$, and is of poor order compared to the $3 \times 3$ case in the monolayer. Though the atomic lattice does not show an increased defect density, the $2 \times 2$ periodicity is often only preserved over a few periods and may even be absent in small patches, compare Fig. 3 (c). In (c) the $2 \times 2$ unit cell and the $\mathrm{TaS}_{2}$ unit cell are indicated. The $2 \times 2$ superstructure is also visible in the Fourier transform of Fig. 3 (c) shown as an inset.

In analogy to the ML, we investigate the BL superstructure with constant height STM on opposing sides of the Fermi level. A comparison of Fig. 3 (d) and (e) illustrates the outof-phase behavior of the CDW contrast (the black and white circles again mark the same positions, but are either local CDW maxima or minima). The poorly ordered $2 \times 2$ thus represents a CDW.

Similar to the ML $3 \times 3 \mathrm{CDW}$, a measurement of the differential conductance shows a reduced LDOS at the Fermi level [cf. Fig. 3 (f)], which is interpreted as a partial CDW gap. The numerical value of the BL gap width is $2 \Delta=(18 \pm 9) \mathrm{meV}$, when the same analysis as for the ML is used.

When interpreting the CDW phase transition from $3 \times 3$ to $2 \times 2$ in going from the ML to the BL, the different environment of the BL compared to the ML has to be taken into account. To specify, the first layer $\mathrm{TaS}_{2}$ growing on $\mathrm{Gr} / \mathrm{Ir}(111)$ has a different substrate (i.e. Gr) than the second layer, which grows on $\mathrm{TaS}_{2}$ as a substrate. Considering the system ML $\mathrm{TaS}_{2} / \mathrm{Gr}$ it can be stated that the interaction between the two layers (and therefore the hybridization) seems negligible, because: (i) In constant current $d I / d V$ maps standing wave patterns can be observed (cf. Supporting Information); (ii) ARPES data are not indicative of any band hybridization. In contrast to this, we assume stronger binding (and therefore stronger hybridization) of the second $\mathrm{TaS}_{2}$ layer to the first one: (I) Standing wave patterns are hardly visible in the BL and are strongly distorted; (II) The epitaxial relation between the second layer and the first layer is strict, while the $\mathrm{ML} \mathrm{TaS}_{2}$ islands display orientation 
scatter with respect to graphene.

Another factor to consider is the possibility of a surplus of Ta atoms being present in the vdW gap between the two $\mathrm{TaS}_{2}$ layers. This phenomenon of self-intercalation has been

observed in bulk $\mathrm{TaS}_{2},{ }^{39,40}$ and may provide additional charge. As we will show in the following, this change in doping level might give rise to a modified CDW periodicity.

Though it should be mentioned that evidence for a $2 \times 2$ ordering has been found in isostructural and -electronic pristine $2 \mathrm{H}-\mathrm{NbSe}_{2},{ }^{41}$ this structure has not yet been observed in $\mathrm{TaS}_{2}$.

\section{Doped Monolayer $\mathrm{TaS}_{2}$}

To investigate the influence of doping on the $\mathrm{CDW}, \mathrm{TaS}_{2} / \mathrm{Gr} / \mathrm{Ir}(111)$ at $500 \mathrm{~K}$ is exposed to Li vapor. The STM topograph in Fig. 4 (a) taken after exposure shows distinct features. (A) On the ML $\mathrm{TaS}_{2}$, a regular superstructure is present, which is enlarged in the inset. Relating this structure to the dense-packed $\mathrm{TaS}_{2}$ directions via the $\mathrm{TaS}_{2}$ lattice allows inference of a $(\sqrt{7} \times \sqrt{7}) R 19.1^{\circ}$ superstructure, which we interpret as a result of ordered Li adatom adsorption. (B) The moiré of $\mathrm{Gr} / \mathrm{Ir}(111)$ is no longer visible on the ML TaS 2 islands. (C) Li intercalation structures can be observed under Gr. These features lead to the interpretation that Li can be found on $\mathrm{TaS}_{2}$, under Gr, and presumably also in between Gr and ML TaS . The indistinct intercalation distribution impedes a precise Li coverage determination. We estimate $0.2 \mathrm{ML}=2.0 \times 10^{18}$ atoms $^{-1}$ as a lower bound to the total $\mathrm{Li}$ coverage. We further performed DFT calculations and estimate an increase of the doping level by 0.25 electrons $(x=-0.25)$ through Li adsorption with a coverage corresponding roughly to the experimental one, see Supporting Material.

The normalized differential conductance around the Fermi level of the $(\sqrt{7} \times \sqrt{7}) R 19.1^{\circ}$ superstructure of $\mathrm{ML} \mathrm{TaS}_{2}$ is shown in Fig. 4 (b). The partial energy gap of size $2 \Delta=(19 \pm 9) \mathrm{meV}$ is strong evidence for a remanent CDW. We note that at the spectroscopy temperature of $5 \mathrm{~K}$ the STM tip is not able to remove Li. Only by increasing the temperature to $30 \mathrm{~K}$, the 

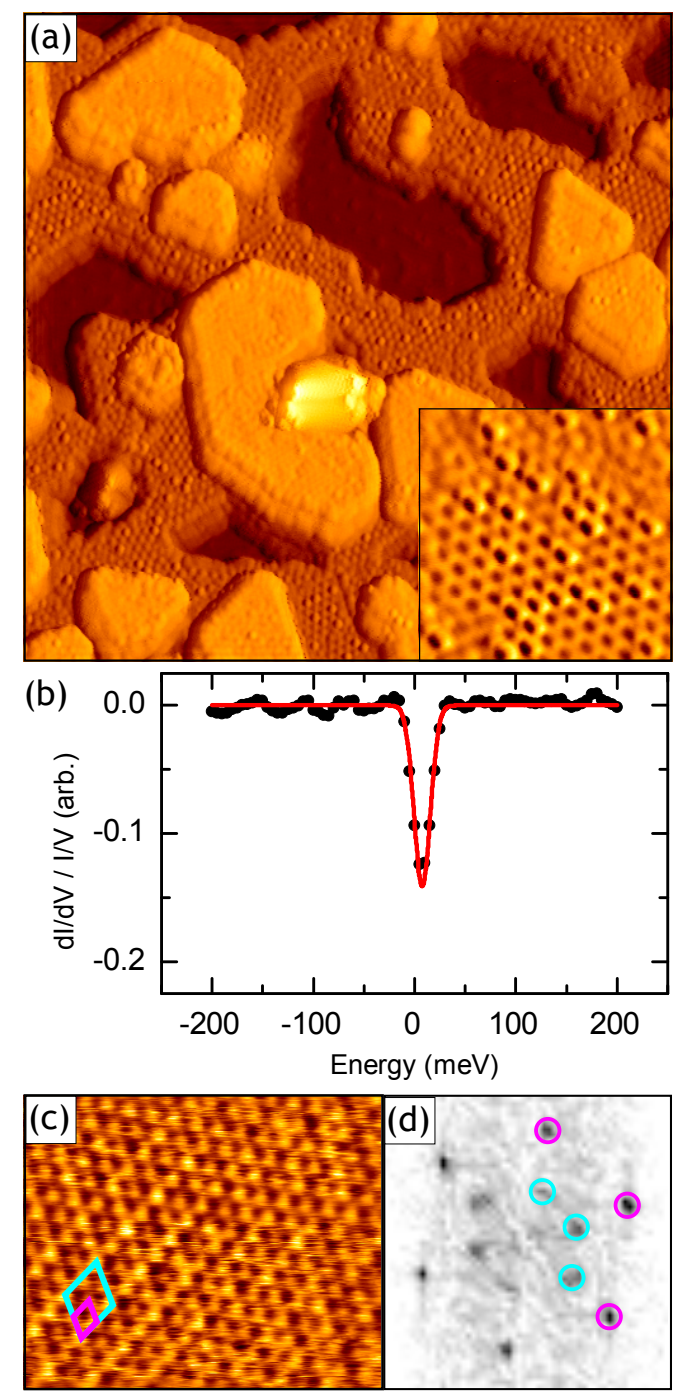

Figure 4: Li doped $\mathrm{TaS}_{2}$ : (a) Large scale STM topograph showing the entire sample surface to be covered by Li adatoms. In the inset an ordered $(\sqrt{7} \times \sqrt{7}) R 19.1^{\circ}$ is visible. (b) Constant height STS spectrum on Li/ML TaS $/$ Gr/Ir(111), treated following the methodology of Ref. 33. The scale is adjusted to be comparable to Fig. 1 (f) and Fig. 3 (f). (c) Constant current STM taken at $30 \mathrm{~K}$ on $\mathrm{ML} \mathrm{TaS}$ showing atomic $\mathrm{TaS}_{2}$ resolution and $2 \times 2$ indications, both marked with their respective unit cell. (d) Corresponding Fourier transformation.

Image information [image size, (stabilization) sample bias, (stabilization) tunneling current]: (a) $60 \times 60 \mathrm{~nm}^{2},-0.8 \mathrm{~V}, 0.1 \mathrm{nA}$, inset: $10 \times 10 \mathrm{~nm}^{2},-0.3 \mathrm{~V}, 0.1 \mathrm{nA}$ (b) $U_{\text {stab }}=-0.15 \mathrm{~V}$, $I_{\text {stab }}=0.2 \mathrm{nA}$, (c) $6 \times 5 \mathrm{~nm}^{2}, 0.01 \mathrm{~V}, 1 \mathrm{nA}$. 
Li-adlayer could be removed from the $\mathrm{ML} \mathrm{TaS}{ }_{2}$ with the STM tip, and the atomic lattice of $\mathrm{TaS}_{2}$ becomes visible. It still exhibits periodic modulations as shown in Fig. 4 (c). Both in the STM topography and in the Fourier transform in Fig. 4 (d) these modulations relate to a poor $2 \times 2$ superlattice ordering.

We also attempted to remove the adsorbed Li by thermal annealing to $900 \mathrm{~K}$. Unexpectedly, this did not result in Li desorption, but in a complete change in the morphology of the $\mathrm{TaS}_{2}$, which is shown in the Supporting Information.

In the literature, bulk $\mathrm{TaS}_{2}$ intercalation compounds show a variety of stable intercalation structures. ${ }^{42}$ Also, ordered Li intercalation structures were reported ${ }^{43,44}$ and even preliminary data for $2 \times 2$ intercalation was mentioned. ${ }^{43}$ It can therefore not be excluded that the observed $2 \times 2$ superlattice may relate to intercalant ordering under ML $\mathrm{TaS}_{2}$. Nevertheless, as no other superstructure is present and a partial gap in the density of states at the Fermi level is found, it is plausible that doping changed the CDW periodicity in the ML from $3 \times 3$ to $2 \times 2$.

\section{Theory}

In order to understand the microscopic origins of the CDW states observed experimentally, we performed calculations of phonon dispersions based on a combination of density-functional theory, density-functional perturbation theory (DFPT) $)^{45,46}$ and many-body perturbation theory. We calculated the phonon self-energy, $\Pi_{\vec{q} \alpha \beta}$ [see Methods, Eq. (2)], which encodes the renormalization of the phonon dispersion due to interactions of the phonons with the electrons from the $\mathrm{TaS}_{2}$ conduction band. On this basis, we analyze here how the interplay of several external control parameters, i.e. electronic hybridization with substrates, doping, and interlayer potentials, affects lattice dynamics and stability in $\mathrm{TaS}_{2}$.

The dependence of the resulting renormalized phonon dispersions in $\mathrm{ML}^{-\mathrm{TaS}_{2}}$ on doping and hybridization with a substrate is shown in Fig. 5 (a). In the quasi-freestanding case, i.e. undoped $(x=0)$ and weakly hybridized $(\Gamma=10 \mathrm{meV})$, the longitudinal-acoustic (LA) 

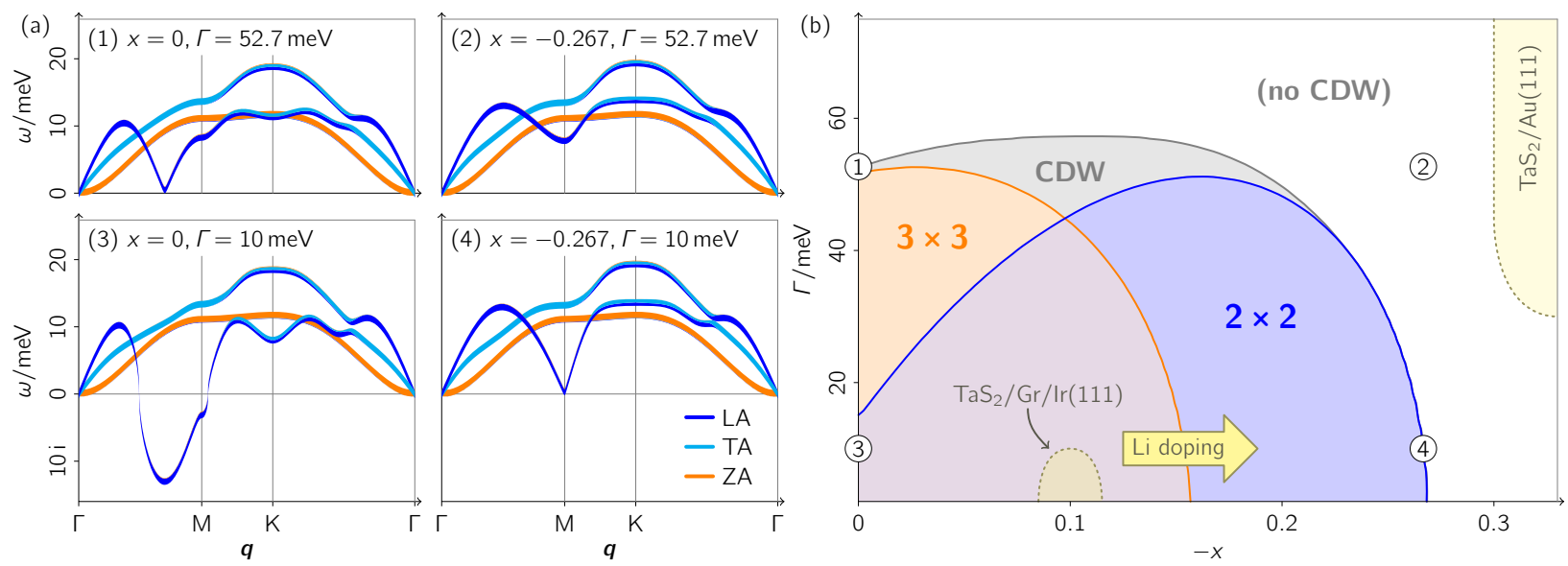

Figure 5: Phonon dispersions and lattice instabilities of $\mathrm{ML} \mathrm{TaS}_{2}$ under different electronic conditions at temperature $T=0$. (a) Acoustic phonon dispersions for different levels of hybridization with the substrate, $\Gamma$ (HWHM of the electronic broadening), and charge doping, $-x$ (in units of electrons per Ta atom). $x<0$ refers to electron addition. The character of the phonon modes, i.e. longitudinal (LA), transverse (TA), or out-of-plane (ZA), is marked in color. Imaginary phonon mode energies indicate that the lattice is unstable towards corresponding periodic lattice distortions. (b) Phase diagram of lattice instabilities in $\mathrm{ML} \mathrm{TaS}_{2}$. The CDW region is defined by the presence of an imaginary phonon energy at one or more $\vec{q}$ points. Regions with instabilities at $M$ for the $2 \times 2$ and at $2 / 3 \Gamma M$ for the $3 \times 3 \mathrm{CDW}$ are marked in color. The experimentally realized situations of pristine ML $\mathrm{TaS}_{2} / \mathrm{Gr} / \mathrm{Ir}(111)$, as well as Li doped as in this work, and ML $\mathrm{TaS}_{2} / \mathrm{Au}(111)$ (Ref. 26,30) are located in the phase diagram. Points corresponding to the phonon dispersions shown in (a) are marked with encircled numbers $1-4$.

phonon branch exhibits a strong Kohn anomaly. Phonon modes with imaginary energy imply that the lattice is unstable with respect to corresponding periodic distortions and charge ordering. The density functional theory establishes a local extremum in the Landau energy functional $E(\xi)=E_{0}+\frac{1}{2} \omega_{\vec{q}}^{2} \xi_{\vec{q}}^{2}+\ldots$. This functional quantifies the energy cost of moving the atoms out of the DFT equilibrium position by some distortion $\xi_{\vec{q}}$. The square of the phonon energy $\omega_{\vec{q}}$ is the coefficient of the quadratic contribution to the energy. Therefore, an imaginary phonon frequency implies an energy gain, which means that the equilibrium is unstable towards distortions with the corresponding wave vector $\vec{q}$. This linear response theory reveals the existence of instabilities, but it does not predict the distorted structure (corresponding to the global minimum of the Landau functional). However, the mode with the largest imaginary frequency, also called leading instability is a clear indicator of energet- 
ically particularly favorable distortions. In the quasi-freestanding case, the instability region extends over large parts of the Brillouin zone including the wave vectors $\vec{q}=2 / 3 \Gamma \mathrm{M}$ and $\mathrm{M}$. At $\vec{q}=2 / 3 \Gamma \mathrm{M}$, which is associated with the $3 \times 3 \mathrm{CDW}$ in the ML, the lattice instability is particularly strong as indicated by a large imaginary phonon frequency.

The doping and hybridization with a substrate can strongly affect the phonon dispersions, as Fig. 5 (a) shows. In essence, we find that electron doping moves the wave vector of the leading instability, i.e. largest imaginary frequency, from $\vec{q} \approx 2 / 3 \Gamma \mathrm{M}$ to $\mathrm{M}$ such that the CDW shifts in wavelength. At electron doping exceeding about 0.27 electrons per $\mathrm{TaS}_{2}$ unit, the lattice becomes dynamically stable, which is in agreement with previous calculations. ${ }^{28}$ An increase in hybridization on the other hand leaves the wavelength of the leading CDW instability largely unchanged, but weakens the Kohn anomaly and eventually also stabilizes the lattice. Thus, both doping and hybridization trigger a quantum phase transition from CDW to undistorted states of the lattice but via different critical wave vectors. The overall relation between lattice instabilities, doping and hybridization is summarized in the phase diagram shown in Fig. 5 (b).

Applied to the experiments performed here, we can state the following: In the ML $\mathrm{TaS}_{2} / \mathrm{Gr} / \operatorname{Ir}(111)$ a doping level of $x=-0.10$ excess electrons per unit cell is measured in ARPES, while no signs of hybridization are experimentally observed. We therefore locate the $\mathrm{ML} \mathrm{TaS}_{2} / \mathrm{Gr} / \operatorname{Ir}(111)$ in the phase diagram of Fig. 5 (b) in the region around $x=-0.10$ and $\Gamma \rightarrow 0$, which is in line with the $3 \times 3$ CDW observed in STM. Intercalating Li increases the doping and moves the experiment towards the right-hand side of the diagram. This is consistent with the experimental observation of a $2 \times 2 \mathrm{CDW}$ in the Li-doped system.

Previous experiments ${ }^{26}$ on $\mathrm{ML} \mathrm{TaS}_{2} / \mathrm{Au}(111)$ did not find any charge order down to 4.7 K. A major difference to the experiments reported here, including those with Li intercalation, is significant hybridization of the $\mathrm{TaS}_{2}$ with the $\mathrm{Au}(111)$ substrate. ${ }^{30}$ Our calculations show that hybridization can be very effective at destabilizing charge order. The hybridiza- 
tion affects the phonon dispersions through the phonon self-energy $\Pi_{\vec{q} \alpha \beta}$, Eq. (2), and is much more effective in quenching $\Pi_{\vec{q} \alpha \beta}$ than thermal broadening (see Methods). For ML $\mathrm{TaS}_{2} / \mathrm{Au}(111)$, the hybridization is energy-dependent ${ }^{30}$ and the half-width at half-maximum (HWHM) broadening $\Gamma$ spans a range between $30 \mathrm{meV}$ and $90 \mathrm{meV}$. We have based our estimate for the electronic broadening on the results of Ref. 30, which contains DFT results for $\mathrm{TaS}_{2}$ with and without $\mathrm{Au}(111)$ substrate. A broadening of $30 \mathrm{meV}$ explains the change in spectral weight at the Van Hove singularity, and a broadening of up to $90 \mathrm{meV}$ is needed to describe the transfer of spectral weight into the gap. Placing $\mathrm{ML} \mathrm{TaS}_{2} / \mathrm{Au}(111)$ in the phase diagram of Fig. 5 (b) we have to account for both the electron (pseudo-)doping ${ }^{30}$ in the range between $x=-0.3^{26}$ and $-0.4^{30}$ and the hybridization between $\Gamma=30 \mathrm{meV}$ and $90 \mathrm{meV}$. Clearly the interplay of both stabilizes the lattice. Lattice relaxation can further support this stabilization. ${ }^{28,29}$

Calculated phonon dispersions of freestanding undoped BL 2H-TaS 2 are shown in Fig. 6 (a). The dispersion and in particular the instability regions in freestanding undoped BL [cf. Fig. 6 (a)] are indeed very similar to the corresponding ML case [cf. Fig. 5 (a)]. Thus, it is a priori unexpected that $\mathrm{BL} \mathrm{TaS}_{2} / \mathrm{Gr} / \mathrm{Ir}(111)$ features a $2 \times 2 \mathrm{CDW}$ in contrast to the $3 \times 3$ $\mathrm{CDW}$ in $\mathrm{ML} \mathrm{TaS}_{2} / \mathrm{Gr} / \mathrm{Ir}(111)$ and also to the $3 \times 3 \mathrm{CDW}$ in bulk $\mathrm{TaS}_{2}$.

What could drive the CDW order in the BL towards $2 \times 2$ ? First, as in the ML case, charge doping could be responsible. Since direct interaction in the bilayer with the substrate is limited to the bottom layer, stronger average charge doping as in the monolayer due to interaction with the substrate is unlikely. A possible cause of stronger average charge doping could be different defect densities in the monolayer and in the bilayer case. Clearly, in the BL the doping from and hybridization with the substrate are naturally different for the bottom and top layer. Also, doping due to defects within the layers can be different for each layer due to inequivalent local growth conditions. Such asymmetries between bottom and top layer might affect CDW formation. In our model, asymmetries of this kind are most simply accounted for in terms of an interlayer bias potential $\Delta \epsilon_{0}$ (energy gain $-\Delta \epsilon_{0} / 2$ or 
penalty $+\Delta \epsilon_{0} / 2$ for an electron residing in the bottom or the top layer), the effect of which is illustrated in Fig. 6 (b) and (c). We find that the interlayer bias can flatten the phonon dispersion in the regions of the instability and eventually also shift the $\vec{q}$ vector of the leading instability from $\vec{q}=2 / 3 \Gamma \mathrm{M}$ towards M. Thus, an effective interlayer bias could also explain the observation of the $2 \times 2 \mathrm{CDW}$ in the $\mathrm{BL}$ regions. Whether additional doping or interlayer asymmetries are the cause for the switching from $3 \times 3$ to $2 \times 2 \mathrm{CDW}$ in the bilayer case in our experiments remains speculative.
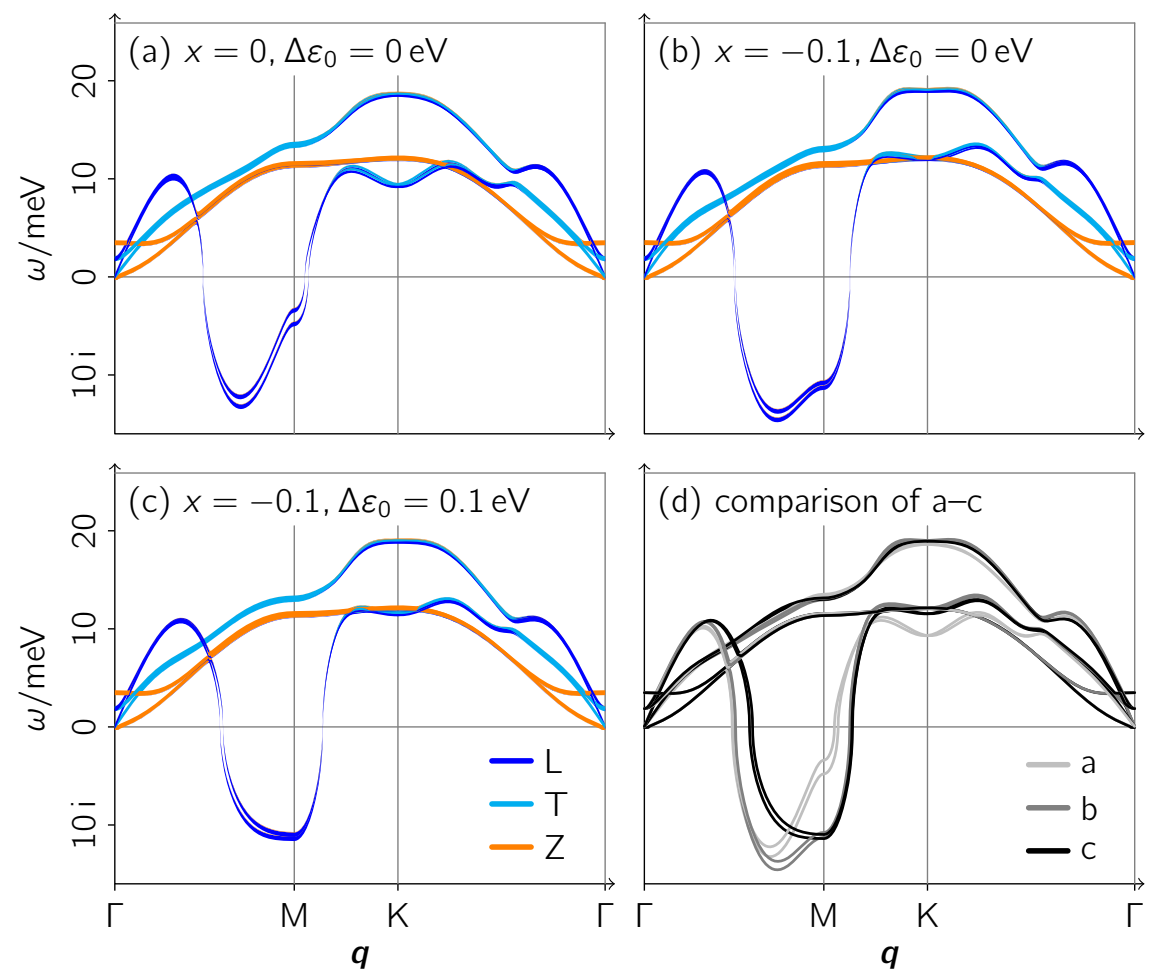

Figure 6: $(\mathrm{a}-\mathrm{c})$ Dispersions of the acoustic phonon branches of $\mathrm{BL} 2 \mathrm{H}-\mathrm{TaS}_{2}$ at different levels of charge doping $x$ and interlayer bias potential $\Delta \epsilon_{0}$ for a fixed broadening $\Gamma=1 \mathrm{meV}$. (d) Direct comparison of the dispersions from panels $(\mathrm{a}-\mathrm{c})$.

\section{Conclusion}

Quasi-freestanding monolayer $1 \mathrm{H}-\mathrm{TaS}_{2}$ on $\mathrm{Gr} / \operatorname{Ir}(111)$ displays a $3 \times 3 \mathrm{CDW}$, while bilayer $2 \mathrm{H}-\mathrm{TaS}_{2}$ on the same substrate exhibits a less well ordered $2 \times 2 \mathrm{CDW}$. The CDW gaps measured are $2 \Delta=(32 \pm 9) \mathrm{meV}$ and $2 \Delta=(18 \pm 9) \mathrm{meV}$, respectively. For the ML, 
the band structure and the FS were determined with ARPES. No indication of hybridization between $\mathrm{Gr}$ and $\mathrm{TaS}_{2}$ is present. Using a TB fit to the ARPES data and the dispersion of states near $\Gamma$ obtained from standing wave patterns in STS maps, a doping of $1.10 \pm 0.02$ electrons per unit cell is deduced.

Exposure to $\mathrm{Li}$ vapor causes a $(\sqrt{7} \times \sqrt{7}) R 19.1^{\circ}$ adatom superstructure on top of monolayer $1 \mathrm{H}-\mathrm{TaS}_{2}$, presumably accompanied by intercalation. A $2 \times 2$ periodicity measured after removal of the adatom phase together with a partial gap of $2 \Delta=(18 \pm 9) \mathrm{meV}$ imply the presence of a $2 \times 2 \mathrm{CDW}$.

Our theoretical analysis reveals the microscopic contributors behind CDW (de-)stabilization and the experimentally observed changes in periodicity. It emphasizes the importance of environmental embedding in the study of $2 \mathrm{~d}$ materials: the theoretically derived CDW phase diagram of $\mathrm{TaS}_{2}$ as a function of doping and hybridization shows that both can suppress CDW order. The critical wave vectors $\vec{q}_{c}$ of the associated quantum phase transition from the CDW to the undistorted lattice depend on the stimulus driving CDW destabilization and are $\vec{q}_{c}=2 / 3 \Gamma \mathrm{M}$, and $\vec{q}_{c}=\Gamma \mathrm{M}$ in the doping and hybridization driven case, respectively.

In the $\mathrm{BL} \mathrm{TaS}_{2}$ case the experimental finding of $2 \times 2$ charge order is surprising given that the phonon dispersion of freestanding pristine $\mathrm{BL} \mathrm{TaS}_{2}$ is very similar to that of the ML. Our analysis shows that an interlayer potential can push the preferential CDW ordering vector towards $\Gamma \mathrm{M}$, which could explain the observed $2 \times 2$ order. Additional charge from self-intercalated $\mathrm{Ta}$ in the vdW gap could be responsible for this phonon renormalization, but this remains speculative.

Quite generally, our results demonstrate that phase diagrams of van der Waals heterostructures are high-dimensional due to the all surface nature of the constituents. Every interface, either between van der Waals bound $2 \mathrm{~d}$ layers or between $2 \mathrm{~d}$ layers and a three-dimensional substrate allows additional control parameters to enter the stage - control parameters that could be made operative in heterostructure based devices. 


\section{Methods/Experimental}

$\mathrm{TaS}_{2} / \mathrm{Gr} / \mathrm{Ir}(111)$ samples are grown in situ as described in detail Ref. 34. In short, the $\operatorname{Ir}(111)$ crystal is cleaned by grazing incidence $4.5 \mathrm{keV} \mathrm{Xe}^{+}$ion exposure and flash annealing to $1500 \mathrm{~K}$. Subsequently, a layer of single-crystalline Gr is grown via temperature programmed growth with ethylene. ${ }^{47}$ The $\mathrm{TaS}_{2}$ growth is performed by $\mathrm{Ta}$ evaporation in a sulfur background pressure, followed by annealing to $1000 \mathrm{~K}$, again in a sulfur background pressure. We measure the resulting coverage in ML, where one ML refers to the one-unit-cell S-Ta-S triple layer, which for $\mathrm{TaS}_{2}$ contains $1.02 \times 10^{19} \mathrm{Ta}$ atoms $\mathrm{m}^{-1}$. Growth conditions are tuned to optimize epitaxy for ARPES or to promote bilayer growth.

The $\mathrm{TaS}_{2}$ layers are checked by low energy electron diffraction and analyzed by STM and ARPES. STM is performed both in a variable temperature $(30 \mathrm{~K}-700 \mathrm{~K})$ STM apparatus and in a low-temperature system at $5 \mathrm{~K}$. If not otherwise indicated, all STM data are taken at $5 \mathrm{~K}$. The software $W S x M^{48}$ was used for STM data processing.

For STS we use standard lock-in technique with modulation frequency of $777 \mathrm{~Hz}$ and modulation amplitudes $V_{\text {mod }}=4 \mathrm{mV}_{\text {rms }}$. For point spectra and constant height topographs $U_{\text {stab }}$ and $I_{\text {stab }}$ of stabilization are indicated.

ARPES spectra were recorded using photons of $h \nu=21.11 \mathrm{eV}$ from a standard He discharge lamp with the sample at $40 \mathrm{~K}$, and synchrotron light at the ANTARES end station of the SOLEIL synchrotron using $50 \mathrm{eV}$ photon energy with the sample at $100 \mathrm{~K}$.

The tight-binding fit was conducted using the quasi-Newton L-BFGS algorithm ${ }^{49}$ to minimize the difference in energy between the ARPES peaks of the d-type $\mathrm{TaS}_{2}$ band and the TB result. The TB model is an adapted version of $\mathrm{ML} \mathrm{MoS}_{2}$ models in literature ${ }^{50}$ as the atomic structure is the same. As a starting point for the fit, $\mathrm{MoS}_{2}$ fit parameters were adopted $^{50}$ with modified on-site energies to shift the Fermi level inside the d-type band.

To determine the doping level, we started from the TB calculated FS, rather than the experimentally measured FS. If one would determine the area of the FS simply from the ARPES map, this would include peak fitting algorithms. As the broadening of the bands 
in the FS can be significant, an accurate fit is difficult if the bands come too close to each other. This reduces the accuracy of the overall area hypothetically determined via this method. The TB model, on the other hand, is a physically reasonable parameter fit that takes into account all ARPES data for the occupied states and the STM data for occupied and unoccupied states. This simplifies the determination of the correct band positions and increases the accuracy of the determined FS area.

A spin-orbit split band in a crystal with $N$ unit cells has $N k$-values, which are uniformly distributed within the first Brillouin zone. Each of the two spin-orbit split subbands has $N$ electron states. In case of the spin-orbit split $d$-derived band in $\mathrm{TaS}_{2}$, the area $A_{\mathrm{FS}, \mathrm{h}, \uparrow}$ of the hole pockets around the $\Gamma$ and $\mathrm{K}$ points for the spin-up subband compared to the area $A_{\mathrm{BZ}}$ of the first Brillouin zone represents thus the degree of band filling of that $d$-state derived subband, i.e. the number of holes $n_{\mathrm{h}, \uparrow}$ per unit cell in that subband. The number of electrons $n_{\mathrm{e}, \uparrow}$ in that subband and per per unit cell is then simply $n_{\mathrm{e}, \uparrow}=1-n_{\mathrm{h}, \uparrow}$. The same procedure is conducted for the $d$-derived spin-down subband. The total number of electrons in the spin-orbit split band is thus

$$
n_{\mathrm{e}}=\left(1-n_{\mathrm{h}, \uparrow}\right)+\left(1-n_{\mathrm{h}, \downarrow}\right)=2-\frac{A_{\mathrm{FS}, \mathrm{h}, \uparrow}+A_{\mathrm{FS}, \mathrm{h}, \downarrow}}{A_{\mathrm{BZ}}}
$$

and amounts to $n_{\mathrm{e}}=1.10 \pm 0.02$.

The goal of our theoretical modeling is to understand the influence of different external stimuli such as hybridization with substrates or electron doping on the $\mathrm{TaS}_{2}$ phonon dispersion and thereby on the lattice instabilities which emerge from the softening of longitudinalacoustic phonons due to metallic screening. To this end, we downfold the electronic structure and lattice dynamics of $2 \mathrm{H}-\mathrm{TaS}_{2}$ to a material-realistic low-energy model containing all phonons and the partially filled electronic conduction band. The phonons entering this model are bare (or, more precisely, partially screened) in the sense that they have to be renormalized due to interactions with electrons from the conduction band. ${ }^{46}$ The corresponding 
bare phonons and the corresponding bare dynamical matrix $D$ are obtained from constrained DFPT,${ }^{46}$ where constrained means that screening within the low-energy electronic subspace is excluded in the determination of the phononic properties. The final experimentally observable phonon dispersion results from the renormalized dynamical matrix $\widetilde{D}=D+\Pi$, which accounts for mutual coupling of the lattice vibrations and the low-energy electronic degrees of freedom through the phonon "self-energy":

$$
\Pi_{\vec{q} \alpha \beta}=\frac{2}{N} \sum_{\vec{k} n m} \widetilde{g}_{\vec{q} \alpha \vec{k} n m} \frac{f\left(\epsilon_{\vec{k}+\vec{q} m}\right)-f\left(\epsilon_{\vec{k} n}\right)}{\epsilon_{\vec{k}+\vec{q} m}-\epsilon_{\vec{k} n}} g_{\vec{q} \beta \vec{k} n m}^{*} .
$$

Here, $\epsilon_{\vec{k}}$ is the electronic energy relative to the Fermi level, $n$ and $m$ label the bands of the low-energy subspace, and $\widetilde{g}$ and $g$ are the fully and partially screened electron-phonon couplings from DFPT and constrained DFPT, respectively. $f\left(\epsilon_{\vec{k} n}\right)$ is the occupation number of the electronic state at wave vector $\vec{k}$ in band $n$.

Based on Eq. (2), we analyze the influence of charge doping, hybridization with substrates, and interlayer bias potentials (BL case) on the phonon dispersions and particularly lattice instabilities of $\mathrm{TaS}_{2}$.

Doping is accounted for, here, in a rigid band model by shifting the band energies $\epsilon_{\vec{k}}$ relative to the Fermi level. Without the substrate, $f$ is the Fermi function which reduces to a step function at $T=0$. Hybridization with the substrate leads to a Lorentzian broadening of the electronic levels and the occupation function at $T=0$ becomes $f(\epsilon)=\frac{1}{2}-\frac{1}{\pi} \arctan \left(\frac{\epsilon}{\Gamma}\right)$, where $\Gamma$ is the half-width at half-maximum (HWHM) of the broadening. This result can be derived under the assumption that the hybridization does not depend on the energy. Note that the arctan decays only polynomially, contrary to the exponential decay of the Fermi-Dirac distribution. This means that states further away from the Fermi level are more relevant for hybridization than for temperature effects.

Our low-energy model is set up in the localized basis of atom centered Wannier functions and atomic displacements. This allows us to also manipulate the model on the level 
of hopping parameters and on-site energies, which helps us study the bilayer. Here, an interlayer-bias potential $\Delta \epsilon_{0}$ is introduced to the bilayer by adding $\Delta \epsilon_{0} / 2$ to all on-site energies of one layer, while subtracting the same value from all on-site energies of the other layer.

All DFT and DFPT calculations have been carried out using QUANTUM ESPRESSO. ${ }^{51,52}$ The modification that is required for constrained DFPT is described in detail in Ref. 46. For the transformation of the electronic energies and electron-phonon coupling to the Wannier basis we used Wannier90 ${ }^{53}$ and the EPW code. ${ }^{54,55}$

We apply the generalized gradient approximation (GGA) by Perdew, Burke and Ernzerhof (PBE) ${ }^{56,57}$ and optimized norm-conserving Vanderbilt pseudo-potentials ${ }^{58}$ from the PseudoDojo pseudo-potential table ${ }^{59}$ at a plane-wave cutoff of 70 Ry. Monkhorst-Pack meshes of $18 \times 18 \vec{k}$ and $6 \times 6 \vec{q}$ points are combined with a Gaussian occupation smearing of $10 \mathrm{mRy}$. Spin-orbit coupling and van-der-Waals interactions are taken into account, the latter via Grimme's DFT-D3 method. ${ }^{60}$

For the ML (BL), assuming a fixed unit cell height of $15 \AA$ ( $25 \AA)$, minimizing the total energy and forces to below $10 \mu \mathrm{Ry} /$ Bohr yields a lattice constant of $3.34 \AA(3.33 \AA$ and a distance of $6.10 \AA$ between the two Ta layers).

For the calculation of the phonon renormalization using Eq. (2), the $\vec{k}$ resolution is increased to $216 \times 216$ points via Wannier/Fourier interpolation of the electronic dispersions and the electron-phonon coupling matrix elements. This ensures convergence also for small values of the broadening.

\section{Author Contributions}

Niels Ehlen and Jan Berges contributed equally to this work. 


\section{Acknowledgement}

This work was funded by the Deutsche Forschungsgemeinschaft (DFG, German Research Foundation) - CRC 1238 (project number 277146847, subprojects A01 and B06), and RTG 2247 - and the European Graphene Flagship. N.E., M.H., J.L., B.S., and A.G. acknowledge the ERC grant no. 648589 'SUPER-2D' and the synchrotron SOLEIL for the allocation of synchrotron radiation beam time. J.B., E.v.L., T.W. thank Ryotaro Arita for providing us with the constrained DFPT source code described in Ref. 46 and the North-German Supercomputing Alliance (HLRN) for computing time.

\section{Supporting Information Available}

The Supporting Information is available free of charge on the ACS Publications website at DOI: .

The Supporting Information includes material which is related to the manuscript and adds additional insight, but is dispensable for the understanding of the main manuscript's issues. As such it includes information on the spatial dependence of the charge density wave amplitude, an example of the differential conductance curve analysis, local density of states maps used for quasi-particle analysis showing standing wave patterns on $\mathrm{TaS}_{2}$, density functional theory calculations estimating the doping of $\mathrm{TaS}_{2}$ through Li adsorption, and document morphological changes after annealing Li doped $\mathrm{TaS}_{2}$. 


\section{References}

1. Wilson, J. A.; Di Salvo, F. J.; Mahajan, S. Charge-Density Waves and Superlattices in the Metallic Layered Transition Metal Dichalcogenides. Adv. Phys. 1975, 24, 117-201.

2. Bednorz, J. G.; Müller, K. A. Possible High $\mathrm{T}_{c}$ Superconductivity in the Ba-La-Cu-O System. Zeitschrift für Phys. B Condens. Matter 1986, 64, 189-193.

3. Kamihara, Y.; Hiramatsu, H.; Hirano, M.; Kawamura, R.; Yanagi, H.; Kamiya, T.; Hosono, H. Iron-Based Layered Superconductor: LaOFeP. J. Am. Chem. Soc. 2006, 128, 10012-10013.

4. Cao, Y.; Fatemi, V.; Demir, A.; Fang, S.; Tomarken, S. L.; Luo, J. Y.; SanchezYamagishi, J. D.; Watanabe, K.; Taniguchi, T.; Kaxiras, E.; Ashoori, R. C.; JarilloHerrero, P. Correlated Insulator Behaviour at Half-Filling in Magic-Angle Graphene Superlattices. Nature 2018, 556, 80-84.

5. Geim, A. K.; Grigorieva, I. V. Van der Waals Heterostructures. Nature 2013, 499, 419425.

6. Novoselov, K. S.; Mishchenko, A.; Carvalho, A.; Neto, A. H. C. 2D Materials and van der Waals Heterostructures. Science 2016, 353, 461.

7. Basov, D. N.; Averitt, R. D.; Hsieh, D. Towards Properties on Demand in Quantum Materials. Nat. Mater. 2017, 16, 1077.

8. Wang, Q.; Li, Z.; Zhang, W.-H.; Zhang, Z.-C.; Zhang, J.-S.; Li, W.; Ding, H.; Ou, Y.-B.; Deng, P.; Chang, K.; Wen, J.; Song, C.-L.; He, K.; Jia, J.-F.; Ji, S.-H.; Wang, Y.Y.; Wang, L.-L.; Chen, X.; Ma, X.-C.; Xue, Q.-K. Interface-Induced High-Temperature Superconductivity in Single Unit-Cell FeSe Films on $\mathrm{SrTiO}_{3}$. Chin. Phys. Lett. 2012, 29, 37402 . 
9. Xi, X.; Zhao, L.; Wang, Z.; Berger, H.; Forró, L.; Shan, J.; Mak, K. F. Strongly Enhanced Charge-Density-Wave Order in Monolayer NbSe 2 . Nat. Nanotechnol. 2015, 10, 765.

10. Yu, Y.; Yang, F.; Lu, X. F.; Yan, Y. J.; Cho, Y.-H.; Ma, L.; Niu, X.; Kim, S.; Son, Y.-W.; Feng, D.; Li, S.; Cheong, S.-W.; Chen, X. H.; Zhang, Y. Gate-Tunable Phase Transitions in Thin Flakes of 1T-TaS 2 . Nat. Nanotechnol. 2015, 10, 270-276.

11. Cao, Y.; Mishchenko, A.; Yu, G. L.; Khestanova, E.; Rooney, A. P.; Prestat, E.; Kretinin, A. V.; Blake, P.; Shalom, M. B.; Woods, C.; Chapman, J.; Balakrishnan, G.; Grigorieva, I. V.; Novoselov, K. S.; Piot, B. A.; Potemski, M.; Watanabe, K.; Taniguchi, T.; Haigh, S. J.; Geim, A. K. et al. Quality Heterostructures from Two-Dimensional Crystals Unstable in Air by Their Assembly in Inert Atmosphere. Nano Lett. 2015, 15, 4914-4921.

12. Ugeda, M. M.; Bradley, A. J.; Zhang, Y.; Onishi, S.; Chen, Y.; Ruan, W.; OjedaAristizabal, C.; Ryu, H.; Edmonds, M. T.; Tsai, H.-Z.; Riss, A.; Mo, S.-K.; Lee, D.; Zettl, A.; Hussain, Z.; Shen, Z.-X.; Crommie, M. F. Characterization of Collective Ground States in Single-Layer $\mathrm{NbSe}_{2}$. Nat. Phys. 2016, 12, 92.

13. Navarro-Moratalla, E.; Island, J. O.; Mañas-Valero, S.; Pinilla-Cienfuegos, E.; Castellanos-Gomez, A.; Quereda, J.; Rubio-Bollinger, G.; Chirolli, L.; SilvaGuillén, J. A.; Agrait, N.; Steele, G. A.; Guinea, F.; van der Zant, H. S. J.; Coronado, E. Enhanced Superconductivity in Atomically Thin $\mathrm{TaS}_{2}$. Nat. Commun. 2016, 7, 11043.

14. Yang, Y.; Fang, S.; Fatemi, V.; Ruhman, J.; Navarro-Moratalla, E.; Watanabe, K.; Taniguchi, T.; Kaxiras, E.; Jarillo-Herrero, P. Enhanced Superconductivity Upon Weakening of Charge Density Wave Transport in $2 \mathrm{H}-\mathrm{TaS}_{2}$ in the Two-Dimensional Limit. Phys. Rev. B 2018, 98, 35203.

15. De La Barrera, S. C.; Sinko, M. R.; Gopalan, D. P.; Sivadas, N.; Seyler, K. L.; Watanabe, K.; Taniguchi, T.; Tsen, A. W.; Xu, X.; Xiao, D.; Hunt, B. M. Tuning Ising Super- 
conductivity With Layer and Spin-Orbit Coupling in Two-Dimensional Transition-Metal Bichalcogenides. Nat. Commun. 2018, 9, 1427.

16. Scholz, G. A.; Singh, O.; Frindt, R. F.; Curzon, A. E. Charge Density Wave Commensurability in $2 \mathrm{H}-\mathrm{TaS}_{2}$ and $\mathrm{Ag}_{x} \mathrm{TaS}_{2}$. Solid State Commun. 1982, 44, 1455-1459.

17. Coleman, R. V.; Giambattista, B.; Hansma, P. K.; Johnson, A.; McNairy, W. W.; Slough, C. G. Scanning Tunnelling Microscopy of Charge-Density Waves in Transition Metal Chalcogenides. Adv. Phys. 1988, 37, 559-644.

18. Wang, C.; Giambattista, B.; Slough, C. G.; Coleman, R. V.; Subramanian, M. A. Energy Gaps Measured by Scanning Tunneling Microscopy. Phys. Rev. B 1990, 42, 8890-8906.

19. Wang, C.; Slough, C. G.; Coleman, R. V. Spectroscopy of Dichalcogenides and Trichalcogenides Using Scanning Tunneling Microscopy. J. Vac. Sci. Technol. B 1991, 9, 10481051.

20. Tonjes, W. C.; Greanya, V. A.; Liu, R.; Olson, C. G.; Molinié, P. Charge-Density-Wave Mechanism in the $2 \mathrm{H}-\mathrm{NbSe}_{2}$ Family: Angle-Resolved Photoemission Studies. Phys. Rev. B 2001, 63, 235101.

21. Tidman, J. P.; Singh, O.; Curzon, A. E.; Frindt, R. F. The Phase Transition in 2H-TaS 2 at 75 K. Philos. Mag. 1974, 30, 1191-1194.

22. Nagata, S.; Aochi, T.; Abe, T.; Ebisu, S.; Hagino, T.; Seki, Y.; Tsutsumi, K. Superconductivity in the Layered Compound $2 \mathrm{H}-\mathrm{TaS}_{2}$. J. Phys. Chem. Solids 1992, 53, $1259-1263$.

23. Bianco Raffaello, Errea Ion, Monacelli Lorenzo, Calandra Matteo, M. F. Quantum Enhancement of Charge Density Wave in $\mathrm{NbS}_{2}$ in the 2D Limit. Nano Lett. 2019, 3, 1-6.

24. Peng, J.; Yu, Z.; Wu, J.; Zhou, Y.; Guo, Y.; Li, Z.; Zhao, J.; Wu, C.; Xie, Y. Disorder Enhanced Superconductivity Toward TaS 2 Monolayer. ACS Nano 2018, 12, 9461-9466. 
25. Wang, Z.; Sun, Y.-Y.; Abdelwahab, I.; Cao, L.; Yu, W.; Ju, H.; Zhu, J.; Fu, W.; Chu, L.; $\mathrm{Xu}, \mathrm{H}$. Surface-Limited Superconducting Phase Transition on 1T-TaS ${ }_{2}$. ACS Nano 2018, 12, 12619-12628.

26. Sanders, C. E.; Dendzik, M.; Ngankeu, A. S.; Eich, A.; Bruix, A.; Bianchi, M.; Miwa, J. A.; Hammer, B.; Khajetoorians, A. A.; Hofmann, P. Crystalline and Electronic Structure of Single-Layer TaS 2 . Phys. Rev. B 2016, 94, 81404.

27. Lin, H.; Huang, W.; Zhao, K.; Lian, C.; Duan, W.; Chen, X.; Ji, S.-H. Growth of Atomically Thick Transition Metal Sulfide Films on Graphene/6H-SiC(0001) by Molecular Beam Epitaxy. Nano Res. 2018, 11, 4722-4727.

28. Albertini, O. R.; Liu, A. Y.; Calandra, M. Effect of Doping on Lattice Instabilities of Single-Layer 1H-TaS, Phys. Rev. B 2017, 95, 235121.

29. Lefcochilos-Fogelquist, H. M.; Albertini, O. R.; Liu, A. Y. Substrate-Induced Suppression of Charge Density Wave Phase in Monolayer 1H-TaS ${ }_{2}$ on $\mathrm{Au}(111)$. Phys. Rev. B 2019, 99, 174113.

30. Shao, B.; Eich, A.; Sanders, C.; Ngankeu, A. S.; Bianchi, M.; Hofmann, P.; Khajetoorians, A. A.; Wehling, T. O. Pseudodoping of a mMetallic Two-Dimensional Material by the Supporting Substrate. Nat. Commun. 2019, 10, 1723-2041.

31. Mattheiss, L. F. Band Structures of Transition-Metal-Dichalcogenide Layer Compounds. Phys. Rev. B 1973, 8, 3719-3740.

32. Meetsma, A.; Wiegers, G. A.; Haange, R. J.; De Boer, J. L. Structure of 2H-TaS2. Acta Crystallogr. C 1990, 46, 1598-1599.

33. Jolie, W.; Knispel, T.; Ehlen, N.; Nikonov, K.; Busse, C.; Grüneis, A.; Michely, T. The Charge Density Wave in $\mathrm{VSe}_{2}$ Revisited. Phys. Rev. B 2019, 99, 115417. 
34. Hall, J.; Pielić, B.; Murray, C.; Jolie, W.; Wekking, T.; Busse, C.; Kralj, M.; Michely, T. Molecular Beam Epitaxy of Quasi-Freestanding Transition Metal Disulphide Monolayers on van der Waals Substrates: A Growth Study. 2D Mater. 2018, 5, 025005.

35. Murray, C.; Jolie, W.; Fischer, J. A.; Hall, J.; van Efferen, C.; Ehlen, N.; Grüneis, A.; Busse, C.; Michely, T. Comprehensive Tunneling Spectroscopy of Quasifreestanding $\mathrm{MoS}_{2}$ on Graphene on $\operatorname{Ir}(111)$. Phys. Rev. B 2019, 99, 115434.

36. Ehlen, N.; Hall, J.; Senkovskiy, B. V.; Hell, M.; Li, J.; Herman, A.; Smirnov, D.; Fedorov, A.; Voroshnin, V. Y.; Di Santo, G.; Petaccia, L.; Grüneis, A.; Michely, T. Narrow Photoluminescence and Raman Peaks of Epitaxial $\mathrm{MoS}_{2}$ on Graphene/Ir(111). $2 D$ Mater. 2018, 6, 11006.

37. Shen, D. W.; Xie, B. P.; Zhao, J. F.; Yang, L. X.; Fang, L.; Shi, J.; He, R. H.; Lu, D. H.; Wen, H. H.; Feng, D. L. Novel mechanism of a Charge Density Wave in a Transition Metal Dichalcogenide. Phys. Rev. Lett. 2007, 99, 216404.

38. Wijayaratne, K.; Zhao, J.; Malliakas, C.; Young Chung, D.; Kanatzidis, M. G.; Chatterjee, U. Spectroscopic Signature of Moment-Dependent Electron-Phonon Coupling in 2H-TaS 2 . J. Mater. Chem. C 2017, 5, 11310-11316.

39. Jellinek, F. The System Tantalum-Sulfur. J. Alloy. Compd. 1962, 4, 9-15.

40. Sakamaki, K.; Funshima, A.; Onuki, Y. Definitive Structure of a Stacking Boundary of Charge Density Waves in $1 \mathrm{~T}-\mathrm{TaS}_{2}$ Using a Scanning Tunneling Microscope. J. Phys. Chem. Solids 1991, 52, 409-418.

41. Chen, C. H. Electron Diffraction Study of the Charge-Density Wave Superlattice in 2H-NbSe 2 . Solid State Commun. 1984, 49, 645-647.

42. Friend, R. H.; Yoffe, A. D. Electronic Properties of Intercalation Complexes of the Transition Metal Dichalcogenides. Adv. Phys. 1987, 36, 1-94. 
43. Thompson, A. H. Electrochemical Studies of Lithium Intercalation in Titanium and Tantalum Dichalcogenides. Phys. B+C 1980, 99, 100-106.

44. Dahn, J. R.; McKinnon, W. R. Lithium Intercalation in 2H-Li $\mathrm{TaS}_{2}$. J. Phys. Condens. Matter 1984, 17, 4231.

45. Baroni, S.; De Gironcoli, S.; Dal Corso, A.; Giannozzi, P. Phonons and Related Crystal Properties from Density-Functional Perturbation Theory. Rev. Mod. Phys. 2001, 73, $515-562$.

46. Nomura, Y.; Arita, R. Ab Initio Downfolding for Electron-Phonon-Coupled Systems: Constrained Density-Functional Perturbation Theory. Phys. Rev. B 2015, 92, 245108.

47. van Gastel, R.; N'Diaye, A. T.; Wall, D.; Coraux, J.; Busse, C.; Buckanie, N. M.; zu Heringdorf, F.-J.; von Hoegen, M.; Michely, T.; Poelsema, B. Selecting a Single Orientation for Millimeter Sized Graphene Sheets. Appl. Phys. Lett. 2009, 95, 121901.

48. Horcas, I.; Fernández, R.; Gómez-Rodríguez, J. M.; Colchero, J.; Gómez-Herrero, J.; Baro, A. M. WSXM: A Software for Scanning Probe Microscopy and a Tool for Nanotechnology. Rev. Sci. Instrum. 2007, 78, 13705.

49. Liu, D. C.; Nocedal, J. On the Limited Memory BFGS Method for Large Scale Optimization. Math. Program. 1989, 45, 503-528.

50. Ridolfi, E.; Le, D.; Rahman, T. S.; Mucciolo, E. R.; Lewenkopf, C. H. A Tight-Binding Model for $\mathrm{MoS}_{2}$ Monolayers. J. Phys. Condens. Matter 2015, 27, 365501.

51. Giannozzi, P.; Baroni, S.; Bonini, N.; Calandra, M.; Car, R.; Cavazzoni, C.; Ceresoli, D.; Chiarotti, G. L.; Cococcioni, M.; Dabo, I.; Dal Corso, A.; de Gironcoli, S.; Fabris, S.; Fratesi, G.; Gebauer, R.; Gerstmann, U.; Gougoussis, C.; Kokalj, A.; Lazzeri, M.; Martin-Samos, L. et al. QUANTUM ESPRESSO: a Modular and Open-Source Soft- 
ware Project for Quantum Simulations of Materials. J. Phys. Condens. Matter 2009, 21, 395502 .

52. Giannozzi, P.; Andreussi, O.; Brumme, T.; Bunau, O.; Nardelli, M. B.; Calandra, M.; Car, R.; Cavazzoni, C.; Ceresoli, D.; Cococcioni, M.; Colonna, N.; Carnimeo, I.; Corso, A. D.; de Gironcoli, S.; Delugas, P.; Jr, R. A. D.; Ferretti, A.; Floris, A.; Fratesi, G.; Fugallo, G. et al. Advanced Capabilities for Materials Modelling with QUANTUM ESPRESSO. J. Phys. Condens. Matter 2017, 29, 465901.

53. Mostofi, A. A.; Yates, J. R.; Pizzi, G.; Lee, Y.-S.; Souza, I.; Vanderbilt, D.; Marzari, N. An Updated Version of wannier90: A Tool for Obtaining Maximally-Localised Wannier Functions. Comput. Phys. Commun. 2014, 185, $2309-2310$.

54. Giustino, F.; Cohen, M. L.; Louie, S. G. Electron-Phonon Interaction Using Wannier Functions. Phys. Rev. B 2007, 76, 165108.

55. Poncé, S.; Margine, E. R.; Verdi, C.; Giustino, F. EPW: Electron-Phonon Coupling, Transport and Superconducting Properties Using Maximally Localized Wannier Functions. Comput. Phys. Commun. 2016, 209, $116-133$.

56. Perdew, J. P.; Burke, K.; Ernzerhof, M. Generalized Gradient Approximation Made Simple. Phys. Rev. Lett. 1996, 77, 3865-3868.

57. Perdew, J. P.; Burke, K.; Ernzerhof, M. Generalized Gradient Approximation Made Simple. Phys. Rev. Lett. 1997, 78, 1396.

58. Hamann, D. R. Optimized Norm-Conserving Vanderbilt Pseudopotentials. Phys. Rev. B 2013, 88, 85117 .

59. van Setten, M. J.; Giantomassi, M.; Bousquet, E.; Verstraete, M. J.; Hamann, D. R.; Gonze, X.; Rignanese, G.-M. The PseudoDojo: Training and Grading a 85 Element 
Optimized Norm-Conserving Pseudopotential Table. Comput. Phys. Commun. 2018, 226, 39-54.

60. Grimme, S.; Antony, J.; Ehrlich, S.; Krieg, H. A Consistent and Accurate Ab Initio Parametrization of Density Functional Dispersion Correction (DFT-D) for the 94 Elements H-Pu. J. Chem. Phys. 2010, 132, 154104. 